\title{
基于局部排序差值细化模式的纹理图像分类
}

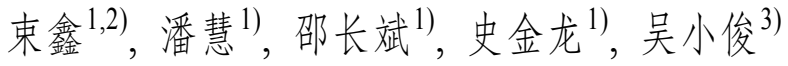 \\ 1) (江苏科技大学计算机学院 镇江 212003) \\ 2) (高维信息智能感知与系统教育部重点实验(南京理工大学) 南京 210094) \\ 3) (江南大学物联网工程学院 无锡 214122) \\ (shuxin@just.edu.cn)
}

\begin{abstract}
摘 要：针对传统局部二值模式及其扩展算法存在特征维度高、不能充分体现局部邻域像素间差值大小信息等问题, 提出一种局部排序差值细化模式(LSDRP). 首先根据采样半径大小对图像进行相应规格的高斯滤波，并按灰度值将 局部邻域采样点排序; 然后计算局部排序邻域内像素间的差值并将其融人排序二值编码对应位置的权值中，从而生 成 LSDRP 特征; 最后选取 LSDRP 特征模式中的高频模式表征图像, 并级联多个半径下 LSDRP 特征的高频模式构成 图像纹理的多尺度表示. 在 Outex, CUReT 和 UMD 纹理库上的实验结果表明, 所提算法计算简单且能在低维度条件 下有效解决纹理分类中存在的光照、旋转变化问题; 特别是在 TC10, TC12_000 和 TC12_001 纹理库上仅需 120 维特 征即可分别达到 $100 \%, 99.38 \%$ 和 $99.72 \%$ 的分类精度.
\end{abstract}

关键词：纹理分类; 局部二值模式; 排序二值模式; 差值细化

中图法分类号: TP391.41

DOI: $10.3724 /$ SP.J.1089.2020.18251

\section{Texture Image Classification Based on Local Sorted Difference Refinement Pattern}

\author{
Shu Xin ${ }^{1,2)}$, Pan Hui ${ }^{1)}$, Shao Changbin ${ }^{1)}$, Shi Jinlong ${ }^{1)}$, and Wu Xiaojun ${ }^{3)}$ \\ 1) (School of Computer Science, Jiangsu University of Science and Technology, Zhenjiang 212003) \\ ${ }^{2)}$ (Key Laboratory of Intelligent Perception and Systems for High-Dimensional Information of Ministry of Education (Nanjing University of Science \\ and Technology), Nanjing 210094) \\ 3) (School of IOT Engineering, Jiangnan University, Wuxi 214122)
}

\begin{abstract}
The traditional local binary pattern and its variants have some shortcomings, such as high feature dimensions, not fully considering the difference between neighboring pixels in local areas and etc. We propose a novel local sorted difference refinement pattern (LSDRP) to overcome the above mentioned deficiencies. Firstly, the texture image is filtered with different Gaussian filters according to the sampling radius, and then the local neighborhood sampling points are sorted according to the pixel value. Secondly, the difference between neighboring pixels in the local area is integrated into the corresponding weight of the sorted binary code for the LSDRP feature generation. Finally, the high-frequency patterns in the LSDRP are selected to represent the texture image and the multi-scale LSDRP feature vector are cascaded to describe the image texture. The experimental results on Outex, CUReT and UMD texture datasets show that the proposed method is simple to calculate and robust to illumination and rotation variant with low-dimensional features.
\end{abstract}

收稿日期: 2020-02-26; 修回日期: 2020-06-30. 基金项目: 国家重点研发计划(2018YFC0309100, 2018YFC0309104); 国家自然科 学基金面上项目 $(61672265 ， 61772244 ， 61876072)$; 高维信息智能感知与系统教育部重点实验室创新基金(JYB201711). 束金金(1979一), 男, 博士, 副教授, 硕士生导师, 主要研究方向为图像理解、模式识别; 潘慧(1996-), 女, 硕士研究生, 主要研究方向为图像理解、 模式识别; 邵长斌(1982一), 男, 硕士, 副教授, 硕士生导师, 主要研究方向为人脸识别、深度学习; 史金龙(1976一), 男, 博士, 教 授, 硕士生导师, 主要研究方向为三维重建、计算机视觉; 吴小俊(1967一), 男, 博士, 教授, 博士生导师, CCF 会员, 主要研究方向 为人工智能、模式识别、计算机视觉. 
It is worth noting that the classification accuracies are $100 \%, 99.38 \%$, and $99.72 \%$ on the TC10, TC12_000, and TC12_001 texture datasets, respectively.

Key words: texture classification; local binary pattern; sorted binary pattern; difference refinement

图像作为人类感知世界的视觉基础，是人们 获取、表达和传递信息的重要手段 ${ }^{[1]}$. 面对海量的 图像数据, 如何让计算机从中挖掘有用信息以完 成图像的分析和理解并提供有效决策成为当前的 研究热点. 而纹理特征作为图像底层特征之一, 在 图像分析、机器视觉和模式识别领域一直占据重要 地位. 无论是对自然图像、医学图像还是遥感图像 而言, 纹理特征的提取和分析都是首要解决的基 本问题 ${ }^{[2]}$. 因此, 如何有效地获取具有表征性的纹 理特征是图像分析和理解的关键所在.

2002 年, Ojala 等 ${ }^{[3]}$ 提出的局部二值模式(local binary pattern, LBP), 因具有计算简单、复杂度低、 线性灰度不变等优点而被广泛用于人脸检测、图像 检索、场景重建等诸多应用领域. 其主要思想在于 引人一种简单的二值量化方式来描述图像局部纹 理结构. 但是, LBP 只反映了图像局部区域中心点 与邻近点像素值间的大小关系, 而忽略了重要的 纹理细节信息 ${ }^{[4]}$. 因而, 在 LBP 的基础上, 众多针 对具体问题的拓展算法被提出. 例如, 在提高抗噪 性能和降低敏感度方面, Tan 等 ${ }^{[5]}$ 提出局部三值模 式(local ternary patterns, LTP), 通过在某一阈值范 围内将局部差分量化为三值模式, 以减少光照和 噪声对特征模式的影响; Liu 等 ${ }^{[6]}$ 基于局部平均的 思想提出二值旋转不变和抗噪声的描述子(binary rotation invariant and noise tolerant, BRINT); 在有 效利用非均匀模式方面, Liao 等 ${ }^{[7]}$ 提出显性 LBP (dominant LBP, DLBP), 通过使用二值模式中出现 频率较高的模式来描述图像纹理, 但其只关注了 图像中各模式的分布, 而忽视了模式类别的重要 信息; Ryu 等 ${ }^{[8}$ 提出排序连续 LBP(sorted consecutive LBP, scLBP), 通过计算二值序列中连续位“ 0 ” 和“1”的数目来编码局部模式, 而不考虑二值序列 中 $0 / 1$ 的跳变次数, 但其需要一个基于 $K \mathrm{D}$-Tree 的 学习阶段来寻找最优参数, 故在提高分类精度的 同时将导致计算量和存储量的增加. 此外，在细化 区分局部模式和融合多种特征方面, Guo 等 ${ }^{[9]}$ 提出 完整的 LBP(completed LBP, CLBP), 通过提取 3 种 具有互补性的描述子，即局部差分的符号、幅值以 及中心像素, 来增强特征的鉴别力; Zhao 等 ${ }^{[10]}$ 在
CLBP 的基础上, 通过丢弃微观结构信息, 直接统 计局部二值序列中 1 的个数提出了完整的局部二 值计数模式(completed local binary count, CLBC), 以实现旋转不变性; Song 等 ${ }^{[11]}$ 通过编码局部采样 点间的强度顺序和非局部结构关系, 提出具有旋 转不变、光照不变以及抗噪声的局部分组序模式 (local grouped order pattern, LGOP)和非局部二值 模式(non-local binary pattern, NLBP).

上述基于 LBP 的改进算法在一定程度上解决 了光照、旋转和噪声等带来的问题, 但缺乏局部像 素间差值大小信息的有效体现; 且随着邻域点的 增加, 所提取的特征维度急剧增长, 尤其是在多特 征融合和多尺度级联时, 从一幅图像中提取的特 征有时高达几千甚至上万维. 而高维特征必将导 致高额的时间和空间消耗, 不利于大数据时代的 图形图像分析, 也不适用于低存储、实时性要求高 的移动设备. 因此, 设计具有较低维度且对旋转、 光照鲁棒的纹理描述子具有十分重要的意义. 本 文通过利用局部像素间差值大小信息提出了一种 低维且有效应对光照、旋转变化的纹理描述子 局部排序差值细化模式(local sorted difference refinement pattern, LSDRP). 首先根据灰度值大小对 局部邻域采样点排序, 得到局部排序二值序列, 同 时计算排序后的邻域采样点和中心点的灰度差值, 以更新局部排序二值序列相应位置处的权值; 然 后分别计算局部排序上部差值细化模式 LSDRP ${ }^{\text {up }}$ 和局部排序下部差值细化模式 LSDRP ${ }^{\text {low }}$, 并将 $\mathrm{LSDRP}^{\mathrm{up}}$ 和 $\mathrm{LSDRP}^{\mathrm{low}}$ 描述子形成的特征直方图级 联, 得到该图像的 LSDRP 特征表示; 最后选取 LSDRP 特征中的高频模式类别表征纹理图像并用 于分类. 此外, 为了降低噪声干扰和增强邻域点与 中心点的关联, 本文提出根据采样半径大小, 对纹 理图像执行相应规格的高斯滤波. 在多个纹理库 上的实验结果表明, 本文算法能够在保持低维度 的同时取得令人满意的分类性能.

\section{1 本文算法}

本文针对传统 LBP 及其扩展算法存在的特征 
维度高、不能充分体现局部邻域像素间差值大小信 息，以及忽略非均匀模式对分类性能的影响等问 题, 提出了 LSDRP 描述子. 利用本文算法提取特 征描述子的过程包括：(1) 根据采样半径大小, 对 图像进行相应规格的高斯滤波, 以获取不同程度 的平滑图像; (2) 在平滑后的图像上提取 LSDRP 描
述子, 以获取纹理图像在不同尺度下的灰度差值 信息; (3) 选择 LSDRP 特征中的高频模式类别表征 纹理图像, 以实现特征的降维; (4) 级联多个采样半 径下, 降维后的 LSDRP 特征, 建立多尺度直方图特 征表示, 以有效融合图像纹理的微观和宏观信息. 图 1 展示了构建多尺度 LSDRP 描述子的框架流程.

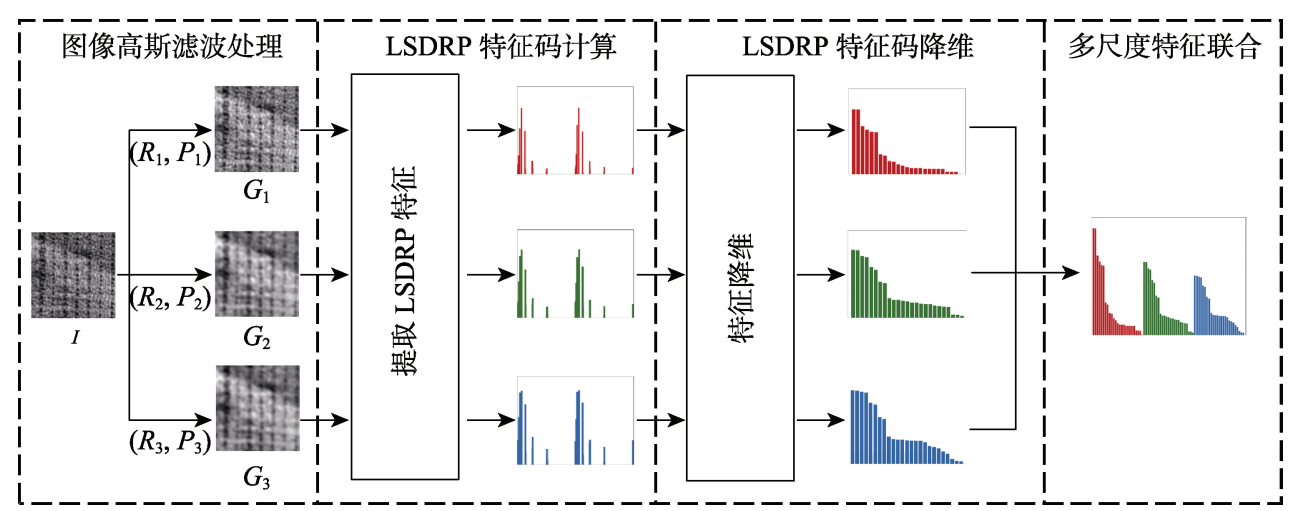

图 1 多尺度 LSDRP 描述子构建框架

\subsection{LSDRP 特征提取}

LBP 为了使定义的特征模式不受光照和对比 度单调变化的影响, 舍弃了弱对比局部模式和强 对比局部模式的区分 ${ }^{[12]}$, 只保留了差值的符号信 息. 因而, LBP 对不同局部邻域中的第 $p$ 位采样点 赋子了相同的权值 $2^{p}$, 且 LBP 较专注于特征模式 的微观结构, 但微观结构信息在旋转不变纹理分 类中是否起主要作用值得探讨. CLBC ${ }^{[10]}$ 对此问题 进行了深人的研究, 并通过实验表明: 用于旋转不 变纹理分类的局部区分信息不是微观结构，而是
局部灰度差值, 这为本文提出的 LSDRP 描述子奠 定了理论基础. LSDRP 描述子通过排序重组了局 部纹理图案的结构, 并将局部像素间的关系分为 邻域值大于或等于中心值的上部和邻域值小于中 心值的下部，再分别将上下部的差值信息融人纹 理特征的编码模式中, 以提高特征的鉴别力. LSDRP 描述子包含 2 个部分, 即局部排序上部差 值细化模式 LSDRP ${ }^{\text {up }}$ 和局部排序下部差值细化模 式 LSDRP ${ }^{\text {low }}$. LSDRP ${ }^{\text {up }}$ 和 LSDRP ${ }^{\text {low }}$ 描述子的详 细提取过程如图 2 所示.

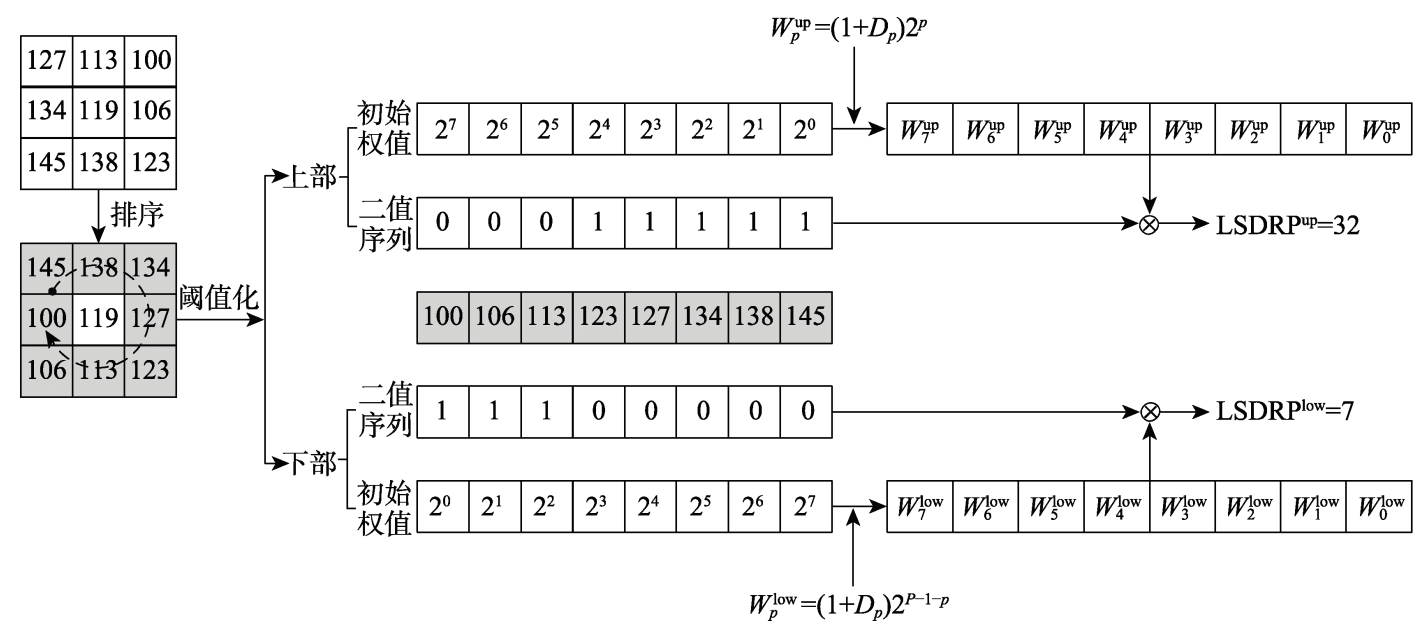

图 $2 \mathrm{LSDRP}^{\mathrm{up}}$ 和 $\mathrm{LSDRP}^{\mathrm{low}}$ 描述子的提取示意

具体步骤如下:

Step 1. 根据灰度值大小, 将图像局部邻域内的采 样点排序, 得到局部排序邻域. 令 $R$ 和 $P$ 分别表示局部
邻域采样半径和采样点数; $g_{\mathrm{c}}$ 表示中心点 $(i, j)$ 处像素 的灰度值; $g_{p}$ 表示以 $g_{\mathrm{c}}$ 为中心, $R$ 为半径的局部邻域 内第 $p$ 位采样点的灰度值; $g_{p}^{\prime}$ 表示排序后的局部邻域 
内第 $p$ 位采样点的灰度值 $(p=0,1, \cdots, P-1)$.

Step2. 在局部排序邻域中, 将采样点和中心点二 值化, 即得到邻域值大于或等于中心值的上部排序二值 序列和邻域值小于中心值的下部排序二值序列, 二值序 列中的 0 和 1 集中分布于两端, 如图 2 所示. 再分别为 上下部二值序列分配初始权值，使得上部对应位置处的 权值为 $2^{p}$, 下部对应位置处的权值为 $2^{P-1-p}$.

Step3. 计算局部排序邻域中采样点和中心点的灰 度差值，并将差值归一化，使其值在 $[0,1]$. 计算公式为

$$
D_{p}=\frac{\left|g_{p}^{\prime}-g_{\mathrm{c}}\right|}{255+g_{\mathrm{c}}}
$$

Step4. 用式(1)分别更新上部和下部排序二值序列 中对应位置处的权值，从而得到包含局部像素间差值大 小信息的新权值为

$$
\begin{gathered}
W_{p}^{\text {up }}=\left(1+D_{p}\right) 2^{p} \\
W_{p}^{\text {low }}=\left(1+D_{p}\right) 2^{P-1-p}
\end{gathered}
$$

Step5. 分别将上部和下部排序二值序列与对应位 置处的新权值 $W_{p}^{\text {up }}$ 和 $W_{p}^{\text {low }}$ 相乘, 再将其累加得到各自的 十进制编码, 分别为

$$
\begin{aligned}
\operatorname{LSDRP}_{R, P}^{\text {up }}(i, j) & =\sum_{p=0}^{P-1} z\left(g_{p}^{\prime}-g_{\mathrm{c}}\right) W_{p}^{\text {up }}, \\
z(x) & =\left\{\begin{array}{l}
1, x \geqslant 0 \\
0, x<0
\end{array} ;\right. \\
\operatorname{LSDRP}_{R, P}^{\text {low }}(i, j) & =\sum_{p=0}^{P-1} f\left(g_{p}^{\prime}-g_{\mathrm{c}}\right) W_{p}^{\text {low }}, \\
f(x) & =\left\{\begin{array}{ll}
0, & x \geqslant 0 \\
1, & x<0
\end{array} .\right.
\end{aligned}
$$

Step6. 对于一幅大小为 $M \times N$ 的纹理图像, 分别 构建 LSDRP $^{\text {up }}$ 和 LSDRP $^{\text {low }}$ 描述子的直方图, 并将 $L_{S D R P}{ }^{u p}$ 和 $L S D R P^{\text {low }}$ 的直方图级联, 由此得到该幅图 像的 LSDRP 特征直方图, 即

$$
\begin{aligned}
& \boldsymbol{H}_{\mathrm{LSDRP}}(k)=\sum_{i=1}^{M} \sum_{j=1}^{N} h\left(\operatorname{LSDRP}_{R, P}^{\text {up }}(i, j), k\right) ; \\
& \boldsymbol{H}_{\text {LSDRP }}(k)=\sum_{i=1}^{M} \sum_{j=1}^{N} h\left(\operatorname{LSDRP}_{R, P}^{\text {low }}(i, j), k\right), 0 \leqslant k \leqslant 2^{P}-1, \\
& h(x, y)=\left\{\begin{array}{l}
1, \text { if } x=y \\
0, \text { else }
\end{array}\right. \\
& \boldsymbol{H}_{\text {LSDRP }}=\left[\boldsymbol{H}_{\text {LSDRPp }^{\text {up }}}, \boldsymbol{H}_{\text {LSDRP }^{\text {low }}}\right] \text {. }
\end{aligned}
$$

对局部邻域采样点排序是为了使特征描述子 具有旋转不变性和相对集中的有效模式(即排序二 值序列中的有效特征模式仅有 $P+1$ 种, 如图 3 所 示). 由式(1)可看到，差值细化的分子部分体现了 局部邻域采样点与中心点的差值大小信息, 分母 部分则融人了该局部区域中心点的灰度值，以便 区分差值相近但中心值大不相同的局部区域. 使 用 $W_{p}^{\text {up }}$ 和 $W_{p}^{\text {low }}$ 更新权值是为了避免直接使用 $D_{p}$
更新权值后导致特征直方图分布较为狭窄的问题， 如图 4 所示. 此外, 为上部和下部排序二值序列分 配图 2 中所示的初始权值. 一方面是为了使初始权 值的分配是与采样点的灰度值相关, 而不是与采 样点的位置相关; 另一方面是为了在差值细化环 节减少模式信息的丢失. 因为加人差值细化后, 对 应位置的权值将大于初始权值, 从而产生部分大 于最大模式编码值 $\left(2^{P}-1\right)$ 的编码, 而大于最大编 码值的特征将会被归到最大编码值中. 因而, 需要 降低大于 $2^{P}-1$ 编码的产生(在 TC12_000 数据集 上, 统计分析了 3 个采样半径下的 LSDRP 特征, 发现图像中大于最大编码值的特征数随着半径的 增大而增多, 大约占总特征数的 $2 \% \sim 6 \%$ ).

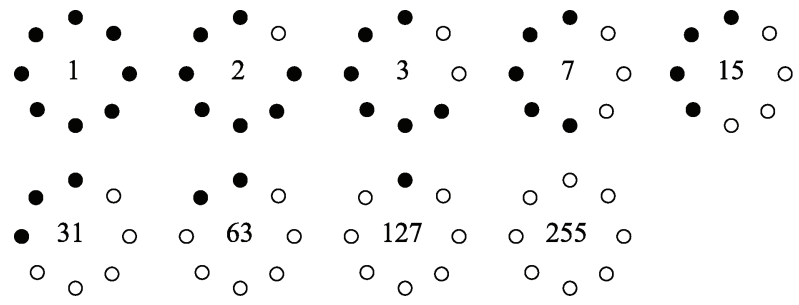

图 $3(1,8)$ 采样模式下排序二值序列中有效特征模式

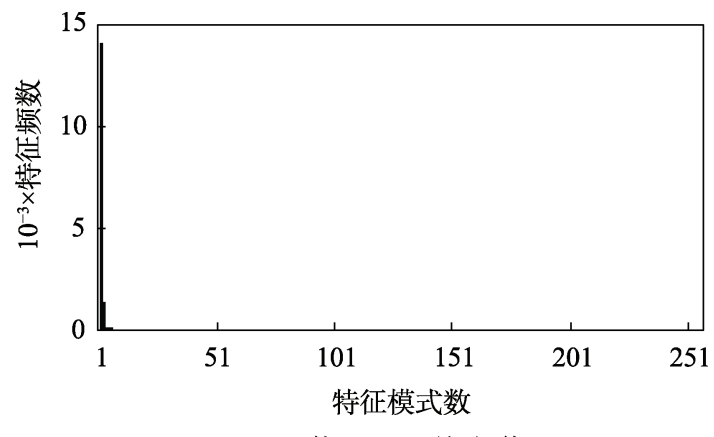

a. 使用 $D^{\text {up 更新权值 }}$

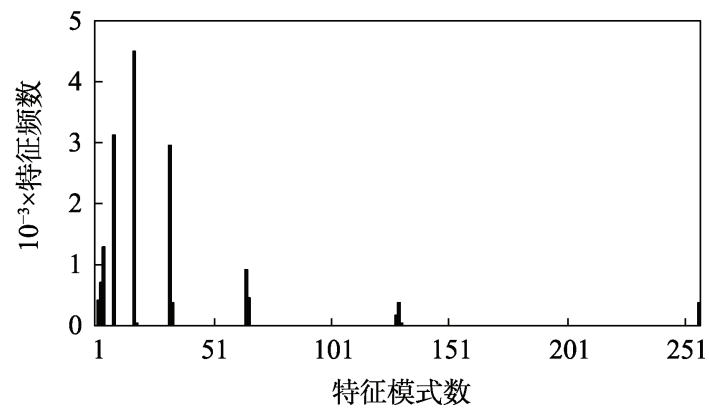

b. 使用 $W^{\mathrm{up}}$ 更新权值

图 $4(1,8)$ 采样模式下更新权值后的 $\mathrm{LSDRP}^{\mathrm{UP}}$ 特征分布

\subsection{LSDRP 特征降维}

LSDRP 描述子包含 LSDRP ${ }^{\text {up }}$ 和 LSDRP ${ }^{\text {low }} 2$ 个 部分, 因而用于表征每幅图像的 LSDRP 特征的维 度为 $2^{P}+2^{P}$. 随着邻域点的增加, 特征维度将急 剧增长, 过高的特征维度将导致高额的时间与空 
间开销且造成各模式间的强相关性 ${ }^{[13]}$. 因此, 本 文需要对 LSDRP 特征降维.

通过减少局部邻域采样点实现特征降维，即 改变一般情况下采样半径和采样点数的关系 $(P=8 R)$, 使得 $R>1$ 时, $P<8 R$. 考虑排序及差值 细化带来的时间开销, 本文固定局部采样点数 $P=8$, 且在固定 $P=8$ 的基础上, 选取特征向量中 最具判别力的特征维度表征纹理图像. 以 LSDRP $^{\text {up }}$ 为例, 仅由排序生成的二值序列中包含 的有效特征模式只有 $(P+1)$ 种, 如图 3 所示. 由于 加人的差值量化函数值域较小, 故每幅图像生成 的 LSDRP ${ }^{\text {up }}$ 特征中具有鉴别力的模式也将分布在
这 $(P+1)$ 种有效特征模式的周边.

在 $\mathrm{TC} 10$ 和 $\mathrm{TC} 12$ 数据集上统计分析 3 个采样 半径下的 LSDRP 特征. 发现 LSDRP 特征中具有鉴 别力的模式的确分布在这 $(P+1)$ 种有效特征模式 周边且只占总特征模式数的较少部分. 如图 5a 图 $5 \mathrm{c}$ 所示, 可以看到, LSDRP 特征直方图中大部分特 征模式的频数趋近于 0 , 基本不具备有效的分类信 息. 为了清楚地观察有效特征的维度, 将图 5a 图 $5 \mathrm{c}$ 中的特征直方图降序排列并保留前 100 维，如 图 5d 图 5f 所示. 可以看到, 有效特征维度基本为 前 40 维, 且随着采样半径的增大, 高频特征频数 降低, 中低频特征频数增加.
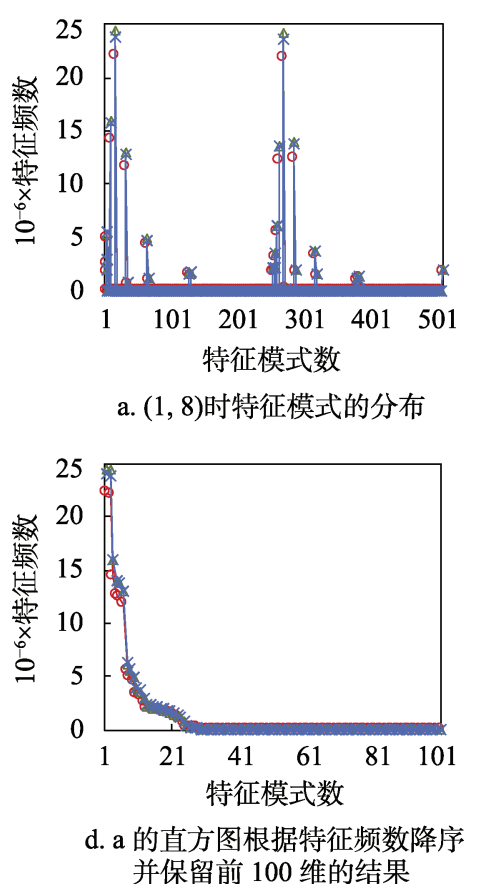

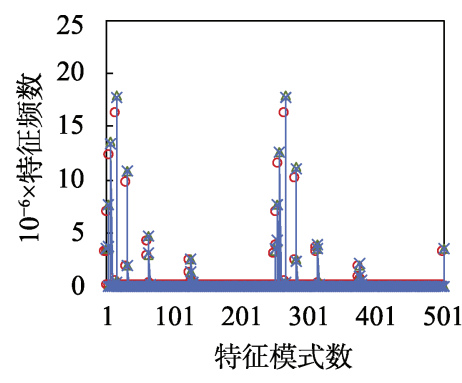

b. (2,8)时特征模式的分布

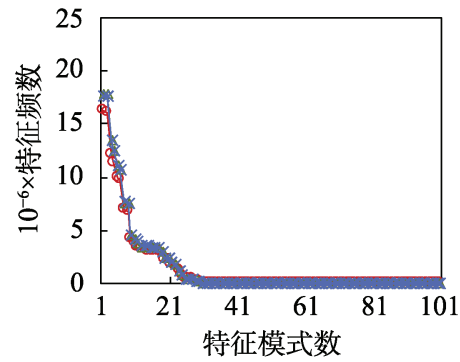

e. b 的直方图根据特征频数降序 并保留前 100 维的结果

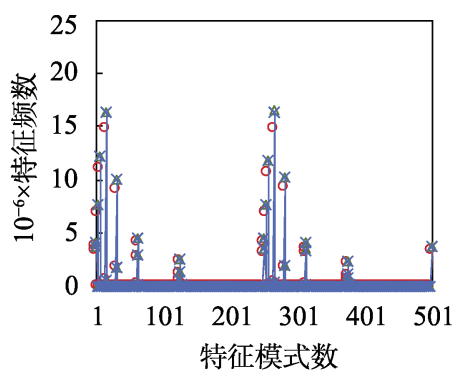

c. $(3,8)$ 时特征模式的分布

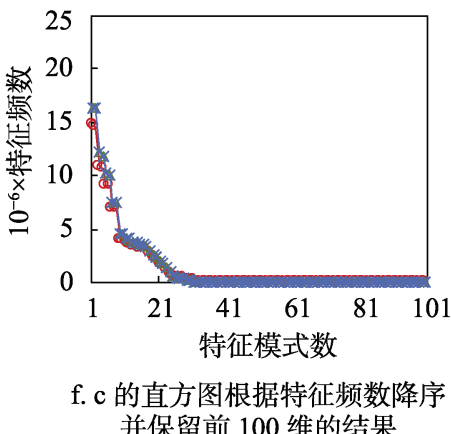
并保留前 100 维的结果

$\longrightarrow \mathrm{TC} 10 ; \multimap \mathrm{TC} 12 \_000 ; \longrightarrow \mathrm{TC} 12 \_001$

图 $5 \mathrm{TC} 10$ 和 $\mathrm{TC} 12$ 数据集在 3 种采样半径下的 LSDRP 特征模式分布

文献[14]证实了保留特征模式类别的策略比 丢弃特征模式类别的策略具有更好的分类性能, 因此在本文中将采取基于经验的方法实现有效特 征模式类别的选取和保留. 具体操作如下:

Step1. 用 $G_{1}, G_{2}, \cdots, G_{n}$ 表示高斯滤波后的训练图像, $n$ 为训练集中图像的总数.

Step2. 计算 $G_{1}, G_{2}, \cdots, G_{n}$ 的 LSDRP 特征直方图，表 示为 $\boldsymbol{H}_{1}, \boldsymbol{H}_{2}, \cdots, \boldsymbol{H}_{n}$, 将所有直方图按模式类别累加, 得 到向量 $\boldsymbol{H}=\boldsymbol{H}_{1}+\boldsymbol{H}_{2}+\cdots+\boldsymbol{H}_{n}$.

Step3．根据模式频数将 $\boldsymbol{H}$ 降序排列得到 $\boldsymbol{H}_{\text {sort }}$ ，保 留 $\boldsymbol{H}_{\mathrm{sort}}$ 的前 $T$ 位高频特征所对应的模式类别.

Step4. 在训练集和测试集中提取每幅图像的
LSDRP 特征, 并从中挑选出 $T$ 种高频模式类别以组成 降维后的特征 $\operatorname{LSDRP}_{T}$, 并用于纹理分类.

\section{2 实验结果与分析}

\section{1 数据库描述与实验设置}

为了合理评价所提出的算法在纹理图像分类 中的性能, 本文使用标准测试协议在 3 个常用的公 共纹理图像数据库 Outex(TC10, TC12_000 和 TC12 001), CUReT 和 UMD 进行了实验, 各纹理 库的相关信息参见文献[15]中的表 2. 在提取特征 前, 先对输人图像进行预处理 ${ }^{[3]}$, 使得每个纹理样 
本的平均强度为 128 , 标准偏差为 20 . 为确保实验 的公平性，所有算法均采用最近邻分类器和卡方 距离进行分类度量. 由于 CUReT 和 UMD 数据库 没有划分固定的训练集和测试集，因此本文在这 2 个数据库上进行了 50 次的分类实验，然后计算平 均分类精度

\section{2 实验参数设定}

\subsection{1 采样半径与高斯滤波}

本文为了降低噪声干扰和增强局部区域中心 点与邻域点间的关联度, 提出在每个尺度获取纹 理特征前先对图像进行高斯滤波平滑操作，且根 据采样半径的大小选取相应的高斯平滑参数. 首 先确定滤波器窗口大小，使得窗口面积和局部区 域面积相同，如 $R=3$ 时，局部区域面积为 $7 \times 7$, 则滤波窗口面积也设为 $7 \times 7$; 然后确定标准差 $\sigma$ 的取值, 由于 $\sigma$ 的大小反映了周围像素对当前像 素的影响程度, 且 $\sigma$ 越大, 远处像素对中心像素 的影响也越大，因此 $\sigma$ 应随着半径的增大而增大. 本文设定的采样半径与高斯滤波参数的对应关系 如表 1 所示.

\subsubsection{LSDRP 特征降维}

本节将分析特征维度对分类性能的影响，以 确定 LSDRP 特征中的高频特征维度. 在 Outex 纹 理库的 TC10，TC12_000 和 TC12_001子库上进行 实验和分析. 在实验中，本文算法的采样半径设为 $R=[1,2,3]$, 采样点数 $P=8$, 采样半径与高斯滤波 对应关系参见表 1. 实验结果如图 6 所示，通过对
表 1 采样半径 $R$ 和高斯滤波参数对应关系

\begin{tabular}{ccc}
\hline \multirow{2}{*}{$R$} & \multicolumn{2}{c}{ 高斯滤波 } \\
\cline { 2 - 3 } & 窗口大小 & $\sigma$ \\
\hline 1 & $3 \times 3$ & 1.0 \\
2 & $5 \times 5$ & 1.5 \\
3 & $7 \times 7$ & 2.0 \\
4 & $9 \times 9$ & 2.5 \\
5 & $11 \times 11$ & 3.0 \\
\hline
\end{tabular}

比每个数据集在 3 个采样半径下分类精度与特征 维度的关系，可以看到当特征维度为 30 40 时，分 类性能达到最佳, 即 40 维以后的特征模式基本不 包含有效信息. 因此, 本文最终确定高频特征模式 数 $T$ 的取值为 40 , 并用 $\mathrm{LSDRP}_{40}$ 表示每幅图像降 维后的特征. 从图 6 中还可以看到，当 $R=2$ 时, 分类精度达到最佳; 当 $R=3$ 时, 分类精度不升反 降, 甚至低于 $R=1$ 时的精度, 这是因为随着半径 的增大, 像素间的相关性逐渐减小. 因此, 在较小 的邻域中即可获得绝大部分的纹理信息 ${ }^{[16]}$. 其次, 半径的增大也将导致局部采样区域变大，低密度 的 8 个采样点无法很好地描述局部区域纹理信息.

\subsection{3 多尺度 LSDRP 特征融合方式}

多尺度特征融合主要有 2 种策略，即半径的连 续选取 (如 $R=[1,2,3]$ ) 和间隔选取 (如 $R=[1,3,5]$ ). 在 Outex(TC10, TC12 000 和 TC12 001), CUReT 和 UMD 纹理库上分析了 2 种特征融合策略对分类 性能的影响, 实验结果如表 2 所示.

可以看到，当半径个数和特征维数取值相同
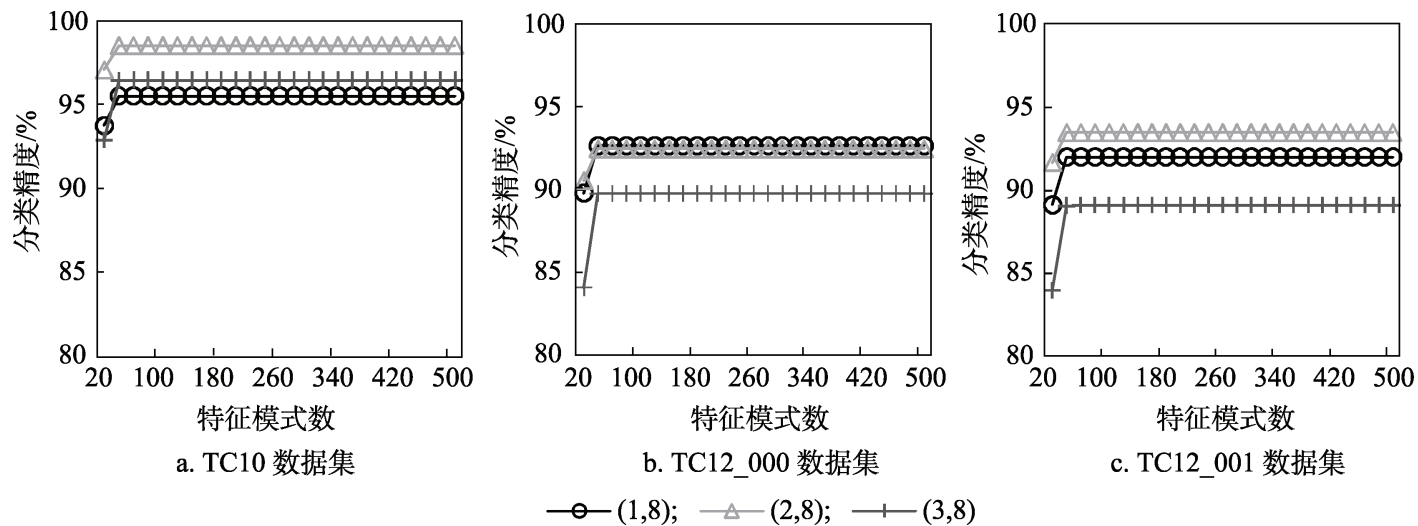

图 6 多个采样半径下的 LSDRP 在 3 个数据集上分类精度随特征维度变化曲线

表 2 特征融合方式对分类性能的影响

\begin{tabular}{lccccc}
\hline \multirow{2}{*}{ LSDRP $_{40(R, P)}$} & \multicolumn{3}{c}{ 分类精度 $/ \%$} \\
\cline { 2 - 5 } & TC10 & TC12_000 & TC12_001 & CUReT & UMD \\
\hline LSDRP $_{40(1,8)}$ & 95.57 & 92.69 & 92.01 & 92.67 & 94.68 \\
LSDRP $_{40(1,8)+(2,8)}$ & 99.43 & 97.62 & 98.10 & 95.73 & 96 \\
LSDRP $_{40(1,8)+(2,8)+(3,8)}$ & 99.87 & 99.10 & 99.14 & 96.55 & 96.21 \\
LSDRP $_{40(1,8)+(3,8)}$ & 99.84 & 99.00 & 99.24 & 96.80 & 96.60 \\
LSDRP $_{40(1,8)+(3,8)+(5,8)}$ & $\mathbf{1 0 0 . 0 0}$ & $\mathbf{9 9 . 3 8}$ & $\mathbf{9 9 . 7 2}$ & $\mathbf{9 7 . 7 3}$ & 96.52 \\
\hline
\end{tabular}

注. 粗体表示每个纹理库上最高的分类精度. 
时，间隔选取的分类精度高于连续选取的分类精 度. 这是由于相邻的采样半径所捕获的特征间存 在较多的冗余信息，而冗余信息的存在会对分类 造成干扰 ${ }^{[17]}$. 因此本文采用间隔选取的策略，及 固定多尺度特征融合时的采样半径 $R=[1,3,5]$.

\subsection{LSDRP 描述子分类性能评估}

\subsection{1 像素灰度差值的有效性评估}

为了验证局部灰度差值信息在旋转不变和光 照不变纹理分类中的有效性，本节将包含差值大 小信息的 LSDRP ${ }^{\text {up }}$ ，LSDRP ${ }^{\text {low }}$ ，LSDRP 以及 $\mathrm{LSDRP}_{40}$ 与不包含差值大小信息的 $\mathrm{CLBP}_{\mathrm{S}}$, $\mathrm{CLBC}_{\mathrm{S}}$ 和 LTP 在 Outex(TC10, TC12_000 和 TC12_001), CUReT 和 UMD 纹理库上进行了单尺 度条件下的比较实验, 并用公开代码实施 $\mathrm{CLBP}_{\mathrm{S}}$, $C L B C$ 和 LTP 算法.

表 3 表 5 给出了不同算法在这几个数据集上 的分类精度. 从中可以看到: (1) 在 5 个数据集上 LSDRP $^{\text {up }}$ 和 LSDRP ${ }^{\text {low }}$ 的分类精度明显低于 LSDRP 和 $\mathrm{LSDRP}_{40}$, 这表明将上下部特征融合的 描述子比仅使用上部或下部的描述子具有更好的 分类鉴别力, 且 $\mathrm{LSDRP}_{40}$ 在大大降低特征维度的 同时能够获得与 LSDRP 相当甚至略高的分类精 度; (2) 在相同 $(R, P)$ 条件下, $\mathrm{LSDRP}_{40}$ 在 5 个数据
集上的分类精度都高于 $\mathrm{CLBP}_{\mathrm{S}}, \mathrm{CLBC}_{\mathrm{S}}$ 和 LTP. 尤 其是在具有光照变化的 TC12_000 和 TC12_001 数 据集上, 相较 LTP 算法, $\mathrm{LSDRP}_{40}$ 的分类精度分别 提升了 $1.72 \% \sim 16.81 \%$ 和 $4.67 \% \sim 18.02 \%$; (3) 当 $R=2, P=8$ 或 $P=16$ 时, 本文算法在 5 个数据集上 仍能取得最高的分类精度; (4) 当 $R=3$ 时, 8 个采样 点的 $\mathrm{LSDRP}_{40}$ 在 5 个数据集上的分类精度略低于 24 个采样点的 LTP, 但当所有算法都使用 8 个采样点 时, $\mathrm{LSDRP}_{40}$ 的分类精度是高于 $\mathrm{CLBP}_{\mathrm{S}}, \mathrm{CLBC}_{\mathrm{S}}$ 以 及 LTP 算法的. 整体而言, 细化灰度差值的 $\mathrm{LSDRP}_{40}$ 算法能够在单尺度以及低密度采样条件 下较好地解决纹理分类中的光照和旋转变化问题.

表 3 相同 $(R, P)$ 条件下不同算法 在 TC10 数据集上的分类精度对比 $\%$

\begin{tabular}{cccccc}
\hline 算法 & $(1,8)$ & $(2,8)$ & $(2,16)$ & $(3,8)$ & $(3,24)$ \\
\hline CLBPs $^{[9]}$ & 84.82 & 84.06 & 89.40 & 86.14 & 95.07 \\
CLBCs $^{[10]}$ & 82.94 & 82.34 & 88.67 & 77.45 & 91.35 \\
LTP $^{\text {riu2[5] }}$ & 94.14 & 94.24 & $\mathbf{9 6 . 9 5}$ & 94.14 & $\mathbf{9 8 . 2 0}$ \\
LSDRP $^{\text {up }}$ & 92.60 & 96.80 & & 93.41 & \\
LSDRP $^{\text {low }}$ & 92.92 & 96.30 & & 92.86 & \\
LSDRP & $\mathbf{9 5 . 5 7}$ & $\mathbf{9 8 . 5 9}$ & & $\mathbf{9 6 . 5 1}$ & \\
LSDRP $_{40}$ & $\mathbf{9 5 . 5 7}$ & $\mathbf{9 8 . 5 9}$ & & 96.48 & \\
\hline
\end{tabular}

注. 粗体表示每个纹理库上最高的分类精度.

表 4 相同 $(R, P)$ 条件下不同算法在 TC12_000 和 TC12_001 数据集上的分类精度对比

$\%$

\begin{tabular}{|c|c|c|c|c|c|c|c|c|c|c|}
\hline \multirow{2}{*}{ 算法 } & \multicolumn{5}{|c|}{ TC12_000 } & \multicolumn{5}{|c|}{ TC12_001 } \\
\hline & $(1,8)$ & $(2,8)$ & $(2,16)$ & $(3,8)$ & $(3,24)$ & $(1,8)$ & $(2,8)$ & $(2,16)$ & $(3,8)$ & $(3,24)$ \\
\hline $\operatorname{CLBPs}^{[9]}$ & 65.46 & 77.92 & 82.26 & 80.07 & 85.04 & 63.68 & 70.65 & 75.20 & 76.67 & 80.78 \\
\hline $\mathrm{CLBCs}^{[10]}$ & 65.02 & 75.16 & 82.57 & 71.88 & 83.82 & 63.17 & 68.59 & 77.41 & 68.56 & 82.75 \\
\hline LTP $^{\text {riu2[5] }}$ & 75.88 & 87.94 & 90.16 & 88.07 & 93.59 & 73.96 & 83.38 & 86.94 & 84.40 & 89.42 \\
\hline LSDRP ${ }^{\text {up }}$ & 87.36 & 90.28 & & 85.30 & & 86.55 & 90.88 & & 83.98 & \\
\hline LSDRP $^{\text {low }}$ & 87.85 & 89.17 & & 85.56 & & 89.38 & 89.68 & & 84.95 & \\
\hline LSDRP & 92.69 & 92.52 & & 89.79 & & 92.01 & 93.50 & & 89.10 & \\
\hline $\mathrm{LSDRP}_{40}$ & 92.69 & 92.52 & & 89.79 & & 92.01 & 93.50 & & 89.07 & \\
\hline
\end{tabular}

注. 粗体表示每个纹理库上最高的分类精度.

表 5 相同 $(R, P)$ 条件下不同算法在 CUReT 和 UMD 数据集上的分类精度对比

\begin{tabular}{|c|c|c|c|c|c|c|c|c|c|c|}
\hline \multirow{2}{*}{ 算法 } & \multicolumn{5}{|c|}{ CUReT } & \multicolumn{5}{|c|}{ UMD } \\
\hline & $(1,8)$ & $(2,8)$ & $(2,16)$ & $(3,8)$ & $(3,24)$ & $(1,8)$ & $(2,8)$ & $(2,16)$ & $(3,8)$ & $(3,24)$ \\
\hline $\mathrm{CLBPs}^{[9]}$ & 80.83 & 82.30 & 85.34 & 79.80 & 87.52 & 92.01 & 91.14 & 92.63 & 88.29 & 92.63 \\
\hline $\mathrm{CLBCs}^{[10]}$ & 78.12 & 76.42 & 79.24 & 66.51 & 80.14 & 91.43 & 89.97 & 93.33 & 84.57 & 92.53 \\
\hline LTP $^{\text {riu2[5] }}$ & 88.15 & 89.44 & 91.37 & 88.81 & 92.78 & 92.24 & 93.82 & 94.99 & 93.74 & 95.54 \\
\hline LSDRP $^{\text {up }}$ & 89.76 & 90.97 & & 89.33 & & 93.13 & 93.22 & & 91.26 & \\
\hline LSDRP $^{\text {low }}$ & 90.09 & 90.74 & & 89.09 & & 92.66 & 92.82 & & 91.15 & \\
\hline LSDRP & 92.93 & 93.34 & & 92.01 & & 94.37 & 95.04 & & 93.65 & \\
\hline $\mathrm{LSDRP}_{40}$ & 92.79 & 93.20 & & 91.87 & & 94.68 & 95.21 & & 93.89 & \\
\hline
\end{tabular}




\subsection{2 与其他典型算法的比较}

为了验证本文算法在纹理分类中的判别力, 该部分将 $\mathrm{LSDRP}_{40}$ 与纹理分类中典型的 $\mathrm{LBP}^{[3]}$, $\mathrm{LTP}^{[5]}, \mathrm{CLBP}^{[9]}, \mathrm{LBPV}^{[18]}, \mathrm{CLBC}^{[10]}, \mathrm{ELBP}^{[19]}, \mathrm{COV}-$ $L B P D^{[20]}$ 和 $\mathrm{BRINT}^{[6]}$ 算法以及近几年提出的 $\mathrm{AMBP}^{[21]}, \mathrm{MRELBP}^{[22]}, \mathrm{FbLBP}^{[23]}, \mathrm{CRDP}^{[24]}$,
$\mathrm{SWMs}^{[25]}, \mathrm{LCCMSP}^{[26]}, \mathrm{PC}-\mathrm{LBP}^{[27]}$ 和 $\mathrm{LGONBP}^{[11]}$

算法在 Outex(TC10，TC12_000 和 TC12_001), CUReT 和 UMD 纹理库上进行了综合比较, 并利用 公开代码实施 LBP, LTP, CLBP, CLBC, 其他算法 的结果均取自对应的原文献和文献[15,24,27], 实 验结果如表 6 所示.

表 6 多尺度级联下 17 种算法在 3 个纹理库上的分类性能对比

\begin{tabular}{|c|c|c|c|c|c|c|c|}
\hline \multirow{3}{*}{ 算法 } & \multicolumn{5}{|c|}{ 分类精度/\% } & \multirow{3}{*}{ 特征维数 } & \multirow{3}{*}{ 文献发表年 } \\
\hline & \multirow{2}{*}{$\mathrm{TC} 10$} & \multicolumn{2}{|c|}{$\mathrm{TC} 12$} & \multirow{2}{*}{ CUReT } & \multirow{2}{*}{ UMD } & & \\
\hline & & 000 & 001 & & & & \\
\hline $\mathrm{LBP}^{\text {riu2[3] }}$ & 97.16 & 88.96 & 83.96 & 93.52 & 94.99 & 54 & 2002 \\
\hline $\operatorname{LTP}^{\text {riu2[5] }}$ & 98.65 & 92.69 & 89.86 & 94.46 & 95.86 & 108 & 2010 \\
\hline $\mathrm{CLBP}^{[9]}$ & 99.17 & 95.23 & 95.58 & 96.94 & 98.88 & 2200 & 2010 \\
\hline LBPV $^{\text {riu2[18] }}$ & 99.27 & 93.92 & 93.92 & 95.85 & 93.79 & 158 & 2010 \\
\hline $\mathrm{CLBC}^{[10]}$ & 99.04 & 94.10 & 95.14 & 96.78 & 98.32 & 1990 & 2012 \\
\hline $\mathrm{ELBP}^{[19]}$ & 99.70 & 98.70 & 97.80 & 96.78 & & 2200 & 2012 \\
\hline COV-LBPD ${ }^{[20]}$ & 98.78 & 95.72 & 97.62 & 94.24 & 92.99 & 289 & 2014 \\
\hline $\mathrm{BRINT}^{[6]}$ & 99.35 & 97.69 & 98.56 & 97.06 & 97.44 & 1296 & 2014 \\
\hline $\mathrm{AMBP}^{[21]}$ & 98.73 & 98.00 & 96.70 & 97.00 & & 320 & 2015 \\
\hline $\operatorname{MRELBP}^{[22]}$ & 99.82 & 99.38 & 99.77 & 97.10 & 98.66 & 800 & 2016 \\
\hline $\mathrm{FbLBP}^{[23]}$ & 99.90 & 98.33 & 98.56 & 97.86 & & 720 & 2017 \\
\hline $\mathrm{CRDP}^{[24]}$ & 99.82 & 98.82 & 98.89 & 98.71 & & 1200 & 2017 \\
\hline $\mathrm{SWMs}^{[25]}$ & 99.56 & 97.85 & 99.12 & 96.86 & & 144 & 2017 \\
\hline $\operatorname{LCCMSP}^{[26]}$ & 99.97 & & & 94.45 & & 2046 & 2018 \\
\hline $\mathrm{PC}-\mathrm{LBP}^{[27]}$ & 100.00 & 98.70 & 99.24 & 97.56 & & 400 & 2018 \\
\hline $\operatorname{LGONBP}^{[11]}$ & 99.48 & 98.45 & 98.89 & 97.73 & 98.28 & 1404 & 2020 \\
\hline $\mathrm{LSDRP}_{40}$ & 100.00 & 99.38 & 99.72 & 97.73 & 96.82 & 120 & \\
\hline
\end{tabular}

注. 粗体表示每个纹理库上最高的分类精度.

根据表 6 中的实验结果，可以得出以下结论:

(1) 在 Outex 纹理库的 TC10, TC12_000 和 TC12_001 子库上, 本文所提算法在特征维度最低 的条件下达到了与最好分类算法 PC-LBP 和 MRELBP 相当甚至更高的分类精度. 尤其是在 $\mathrm{TC} 10$ 数据集上, $\mathrm{LSDRP}_{40}$ 获得了与 PC-LBP 相同 的最高分类精度 $100 \%$ ，达到了完美匹配的效果． 在 TC12_000 和 TC12_001 数据集上, $\mathrm{LSDRP}_{40}$ 也 分别取得了第 1 和第 2 的分类精度, 仅略低于最好 算法 MRELBP 分类精度的 $0.05 \%$. 值得注意的是， PC-LBP 和 MRELBP 算法的复杂度远高于本文算 法, 且 MRELBP 描述子是在获取高密度邻域采样 点中值的基础上, 由 3 个不同类别的操作符构成. 因而，在 Outex 纹理库上的总体分类性能表明本文 算法能够在低维度条件下简单有效地解决纹理分 类中的光照、旋转变化问题.

(2) 在 CUReT 纹理库上，本文算法的分类精
度位列前 3 , 略低于 CRDP 和 FbLBP算法. 在 UMD 纹理库上, 本文算法的分类精度位于中等水平，但 相较于具有最高分类精度的 CLBP 而言, $\mathrm{LSDRP}_{40}$ 的特征维度降低了 18 倍. 在 CUReT 和 UMD 纹理 库上分类性能欠佳的主要原因是: 这 2 个纹理库都 具有尺度和视点变化, 而 $\mathrm{LSDRP}_{40}$ 描述子是针对 旋转和光照变化提出的; $\mathrm{LSDRP}_{40}$ 仅由一种类型 的描述子组成, 因而在面对具有尺度和视点变化 的纹理库时处理能力有限.

(3) 从分类精度、计算复杂度和特征维度 3 方 面考虑，本文算法具有显著优势.

\subsection{LSDRP 时效性分析}

为了验证本文算法在纹理分类中的时效性, 该部分将 $\mathrm{LSDRP}_{40}$ 与 $\mathrm{LBP}, \mathrm{LTP}, \mathrm{CLBP}, \mathrm{CLBC}$, BRINT 和 MRELBP 进行了比较. 实验平台为 MATLAB R2017b，PC 配置为 Intel(R) Xeon(R) Silver 4110 CPU @ 2.10 GHz 和 128 GB RAM. 表 7 
给出了多尺度级联时不同算法在 $\mathrm{TC} 10$ 数据集上提 取一幅图像特征所需的平均时间和特征维度. 可以 看出：(1) LBP 的特征提取时间最短; (2) $\mathrm{LSDRP}_{40}$ 的提取时间与 LTP, CLBP 和 CLBC 相近; (3) MRELBP 的特征提取时间最长, 大约为本文算法 提取时间的 6 倍. 这是由于 $\mathrm{LSDRP}_{40}$ 使用低密度的 采样点数 $(P=8)$, 弥补了特征提取过程中由排序、 差值细化以及高斯滤波带来的时间开销. 因此, 本 文算法能够在获取较高分类精度的同时具有计算 高效的优点.

表 7 不同算法在 TC10 数据集上提取特征的时效性

\begin{tabular}{lcr}
\hline \multicolumn{1}{c}{ 算法 } & 时间/s & 特征维度 \\
\hline $\mathrm{LBP}^{\mathrm{riu2}[3]}$ & 0.019 & 54 \\
$\mathrm{LTP}^{\mathrm{riu} 2[5]}$ & 0.028 & 108 \\
$\mathrm{CLBP}^{[9]}$ & 0.031 & 2200 \\
$\mathrm{CLBC}^{[10]}$ & 0.025 & 1900 \\
$\mathrm{BRINT}^{[6]}$ & 0.063 & 1296 \\
$\mathrm{MRELBP}^{[22]}$ & 0.175 & 800 \\
$\mathrm{LSDRP}_{40}$ & 0.028 & 120 \\
\hline
\end{tabular}

\section{3 结 语}

本文主要针对原始 LBP 及其扩展算法存在的 特征高维度以及不能充分体现局部邻域像素间差 值大小信息等问题，提出了多尺度 LSDRP 算法. 根据采样半径大小对图像进行相应尺度的高斯滤 波，以获得图像在多尺度下的纹理特征；根据灰度 值大小对局部邻域采样点排序, 使得纹理描述子 在不使用旋转不变模式的条件下具有高效的旋转 鲁棒性; 将邻域采样点和中心点的灰度差值信息 融人纹理特征的编码模式中, 并分为 LSDRP ${ }^{\text {up }}$ 和 $\mathrm{LSDRP}^{\text {low }} 2$ 个部分, 不仅增强了特征的健壮性, 还有效地表征了局部区域的灰度差值信息; 通过 寻找 LSDRP 特征中的高频模式类别表征纹理图 像，在实现特征低维度的同时有效避免了低频模 式对纹理分类性能的影响. 在 3 个纹理库上的实验 结果表明，本文算法能够在保持低维度的同时很 好地解决纹理分类中存在的光照和旋转变化问题, 且能够在低密度采样条件下有效捕获局部像素间 的纹理信息. 尤其是在 TC10, TC12_000 和 TC12_001 数据集上仅需 120 维的特征即可达到 $100 \%, 99.38 \%$ 和 $99.72 \%$ 的分类精度. 此外, 本文 算法在具有视点和尺度变化的纹理库上分类性能 欠佳，在后期的工作中将重点关注这一问题，并通 过改进和探寻新的方法来改善不足.

\section{参考文献(References):}

[1] Wang Kai. Image de-noising and area identification based on wavelet analysis[D]. Chengdu: Southwest Jiaotong University, 2009(in Chinese)

(王凯. 基于小波分析的图像去噪及区域识别[D]. 成都: 西 南交通大学, 2009)

[2] Liu Li, Zhao Lingjun, Guo Chengyu, et al. Texture classification: state-of-the-art methods and prospects[J]. Acta Automatica Sinica, 2018, 44(4): 584-607(in Chinese) (刘丽, 赵凌君, 郭承玉, 等. 图像纹理分类方法研究进展和 展望[J]. 自动化学报, 2018, 44(4): 584-607)

[3] Ojala T, Pietikainen M, Maenpaa T. Multiresolution gray-scale and rotation invariant texture classification with local binary patterns[J]. IEEE Transactions on Pattern Analysis and Machine Intelligence, 2002, 24(7): 971-987

[4] Shu Xin, Tang Hui, Yang Xibei, et al. Research on face anti-spoofing algorithm based on DQ_LBP[J]. Journal of Computer Research and Development, 2020, 57(7): 1508-1521(in Chinese)

(束金鍂，唐慧，杨习贝，等. 基于差分量化局部二值模式的人 脸反欺计算法研究 [J]. 计算机研究与发展, 2020, 57(7): 1508-1521)

[5] Tan X Y, Triggs B. Enhanced local texture feature sets for face recognition under difficult lighting conditions[J]. IEEE Transactions on Image Processing, 2010, 19(6): 1635-1650

[6] Liu L, Long Y L, Fieguth P W, et al. BRINT: binary rotation invariant and noise tolerant texture classification[J]. IEEE Transactions on Image Processing, 2014, 23(7): 3071-3084

[7] Liao S, Law M W K, Chung A C S. Dominant local binary patterns for texture classification[J]. IEEE Transactions on Image Processing, 2009, 18(5): 1107-1118

[8] Ryu J, Hong S, Yang H S. Sorted consecutive local binary pattern for texture classification[J]. IEEE Transactions on Image Processing, 2015, 24(7): 2254-2265

[9] Guo Z H, Zhang L, Zhang D. A completed modeling of local binary pattern operator for texture classification[J]. IEEE Transactions on Image Processing, 2010, 19(6): 1657-1663

[10] Zhao Y, Huang D S, Jia W. Completed local binary count for rotation invariant texture classification[J]. IEEE Transactions on Image Processing, 2012, 21(10): 4492-4497

[11] Song T C, Feng J, Luo L, et al. Robust texture description using local grouped order pattern and non-local binary pattern[J]. IEEE Transactions on Circuits and Systems for Video Technology, 2020, 2(99): 1

[12] Satpathy A, Jiang X D, Eng H L. LBP-based edge-texture features for object recognition[J]. IEEE Transactions on Image Processing, 2014, 23(5): 1953-1964

[13] Geng Lichuan, Cheng Yun, Su Songzhi, et al. RBFD: a robust image local binary feature descriptor[J]. Journal of Computer-Aided Design \& Computer Graphics, 2015, 27(5): 815-823(in Chinese)

(耿利川, 成运, 苏松志, 等. RBFD: 一种鲁棒的图像局部二 值特征描述子 [J]. 计算机辅助设计与图形学学报, 2015, 27(5): 815-823)

(下转第 1966 页) 